\title{
APLICATIVOS MÓVEIS (APPS) PARA ANALISAR INTERAÇÕES MEDICAMENTOSAS NO TRATAMENTO DE HIV/AIDS: UMA REVISÃO SISTEMÁTICA EM PLATAFORMAS DIGITAIS
}

\author{
Daniela Bicalho ${ }^{1}$, Tácio de Mendonça Lima². \\ ${ }^{1}$ Universidade de São Paulo, ${ }^{2}$ Universidade Federal Rural do Rio de Janeiro \\ E-mail para correspondência: tacioml@gmail.com
}

Submetido em: 11/06/2021 e aprovado em: 02/08/2021

\begin{abstract}
RESUMO
Introdução: O número de aplicativos móveis aumentou nos últimos anos, dificultando a escolha de apps úteis e de qualidade. Desta forma, o objetivo do estudo foi identificar a disponibilidade e a qualidade dos apps para analisar PIM no tratamento de HIV/AIDS. Métodos: Foi realizada uma busca nas lojas Google Play Store e Apple Store, utilizando a palavra-chave "HIV drug interaction". O instrumento Mobile App Rating Scale (MARS) foi utilizado para avaliação da qualidade dos aplicativos. Resultados e Discussão: Cinco aplicativos atenderam aos critérios de inclusão. A maioria dos aplicativos estava disponível na Google Play Store, desenvolvidos por instituições hospitalares e atualizados nos últimos 2 anos. As pontuações médias de avaliação objetiva e subjetiva do instrumento MARS foram de 3,52 (DP = 0,65) e 3,00 (DP = 0,84), respectivamente. Apenas um aplicativo foi classificado com boa qualidade. Conclusão: O estudo encontrou lacunas na qualidade destes aplicativos, sendo importante utilizá-los com cautela na prática clínica.
\end{abstract}

Palavras-chave: HIV; aplicativos móveis; smartphone; interações medicamentosas; revisão

\begin{abstract}
Introduction: Mobile applications (apps) increase in recent years, making it difficult to choose useful and quality apps. Thus, this review is aimed to identify apps to check drug interactions in HIV treatment and perform a quality assessment. Methods: A comprehensive search was conducted in Google Play Store and Apple Store using search term "HIV drug interaction". The Mobile App Rating Scale was used to quality assessment. Results and Discussion: Five apps fully met the eligibility criteria. Most applications were available in Google Play Store, developed by hospital institutions and were updated within the last two year. The average scores for objective and subjective MARS quality of these applications were $3.52(\mathrm{SD}=0.65)$ and $3.00(\mathrm{SD}=0.84)$, respectively. Only one app was considered with good quality. Conclusion: This review observed gaps in quality of the apps, being important using these apps with cautiously in clinical practice.
\end{abstract}

Keywords: HIV; smartphone; mobile applications; drug interactions; review 


\section{INTRODUÇÃO}

As doenças crônicas, transmissíveis ou não transmissíveis, constituem um problema de saúde pública mundial com custos diretos e indiretos em relação à saúde dos afetados bem como dos sistemas de saúde. ${ }^{(1)}$ Dentre as doenças crônicas transmissíveis, a infecção pelo vírus da imunodeficiência humana (HIV), causador da síndrome de imunodeficiência adquirida (AIDS) tem grande prevalência em nossa sociedade. ${ }^{(2)}$

A infecção pelo HIV atinge principalmente os linfócitos T CD4+ responsáveis pela defesa do organismo e está associada as relações sexuais sem preservativos, transfusões sanguíneas não seguras, uso coletivo de seringas, instrumentos perfurocortantes contaminados e transmissões verticais. Os aspectos clínicos da infecção pelo HIV ser dividida em quatro fases: 1) infecção aguda; 2) fase assintomática, também conhecida como latência clínica; 3) fase sintomática inicial ou precoce; e 4) AIDS. As primeiras manifestações clínicas são parecidas com um quadro gripal, porém doenças oportunistas como hepatites virais, tuberculose, pneumonia, toxoplasmose e alguns tipos de câncer podem ocorrer no seu estágio mais avançado. $^{(3)}$

De acordo com Organização Mundial da Saúde (OMS), em 2018, havia 37,9 milhões de pessoas viviam com infecção pelo HIV no mundo, representando 0,5\% da população. Embora desigualmente distribuída em todo o mundo, nas regiões africanas vivem mais de dois terços da população afetada. Entre 2000 e 2018, as novas infecções por HIV diminuíram 37\% e as mortes relacionadas ao HIV caíram 45\%, com 13,6 milhões de vidas salvas devido à terapia antirretroviral. $^{(4)}$

Em 2016, a OMS adotou a Estratégia do Setor Global de Saúde para HIV, que exige sua eliminação como ameaça à saúde pública até 2030. A meta é que $90 \%$ das pessoas que vivem com HIV sejam testadas, $90 \%$ tratadas e $90 \%$ do vírus suprimidos. Até 2030 , os países e as regiões precisam reduzir novas infecções (incidência) para menos de 500.000, zero novas infecções entre os bebês, e as mortes (mortalidade) em menos de 500.000. As intervenções primárias foram projetadas como estratégias para eliminar o HIV até 2030, como acesso e uso racional do tratamento do antirretroviral. ${ }^{(5)}$

As terapias antirretrovirais (TARV) permitiram um controle na progressão da infecção pelo HIV, uma vez que contribui para a diminuição da morbimortalidade e para o aumento da expectativa de vida dos pacientes infectados em resultado da inibição da replicação viral e, consequente elevação dos níveis de linfócitos T CD4+. ${ }^{(6,7)}$ Todavia, o uso destes medicamentos associado a outros aumentam os riscos de potenciais interações medicamentosas (PIM), em 
decorrência da polifarmácia, podendo levar a toxicidade ou diminuindo a efetividade dos tratamentos. ${ }^{(8,9)}$ Portanto, é fundamental o conhecimento dos profissionais de saúde em relação às PIM em pacientes submetidos ao tratamento antirretroviral, bem como os médicos devem considerar as características dos medicamentos e seus efeitos adversos, suas vias metabólicas e, especialmente, as possibilidades de interações. ${ }^{(10,11)}$

No mundo globalizado, a tecnologia móvel apresenta uma oportunidade de auxiliar profissionais no cuidado em saúde. Nos últimos anos, o smartphone tem sido uma das invenções mais prósperas, revolucionando e facilitando a assistência a saúde. ${ }^{(12)} \mathrm{O}$ rápido crescimento de aplicativos móveis (apps) médicos e de saúde para smartphones demonstra que os desenvolvedores veem um mercado atual e promissor, oferecendo benefícios como a praticidade. ${ }^{(10)}$ Há um número crescente de profissionais de saúde que usam smartphones em diferentes áreas, destacando o monitoramento remoto, o apoio ao diagnóstico e o apoio a tomada de decisão. ${ }^{(13)}$ Estudos demonstraram que o uso de apps médicos por profissionais de saúde na prática clínica diária variou de $74 \%$ a $87 \% .^{(14,15)}$

Aplicativos móveis na área da saúde aumentaram exponencialmente nos últimos anos. Estima-se que 40.000 apps relacionados à saúde estejam disponíveis em todas as principais lojas de apps. Entretanto, o grande número de aplicativos médicos confunde os profissionais de saúde na escolha dos apps considerados úteis e confiáveis para uso na prática clínica. ${ }^{(16,17)}$ Para minimizar este problema, alguns estudos revisaram a qualidade de apps em diferentes contextos utilizando instrumentos validados, como na adesão a medicamentos ${ }^{(18)}$, informações sobre medicamentos $^{(19)}$, hipertensão ${ }^{(20)}$ e epilepsia ${ }^{(21)}$. Entretanto, nenhum estudo avaliou apps para verificar PIM no tratamento de HIV/AIDS.

Assim, esta revisão tem por objetivo identificar a disponibilidade de aplicativos móveis para analisar PIM no tratamento de HIV/AIDS, bem como avaliar criticamente a qualidade destes apps.

\section{MÉTODOS}

Desenho do estudo

Trata-se de uma revisão sistemática em plataformas digitais a fim de identificar a disponibilidade de aplicativos móveis para analisar PIM no tratamento de HIV/AIDS. 
Estratégia de pesquisa

Foi realizada uma busca abrangente até fevereiro de 2021 nas lojas Google Play Store (Android) e Apple Store (iOs) para identificar apps relevantes sobre o tema. A estratégia de busca incluiu o termo "HIV drug interaction" em ambas as lojas de apps. Aplicativos duplicados ou irrelevantes, como jogos, foram eliminados.

\section{Seleção dos aplicativos móveis}

Para este estudo foram incluídos os apps que atendessem os seguintes critérios: 1) relacionado especificamente com PIM no tratamento de HIV/AIDS; 2) para profissionais de saúde; 3) na língua inglesa, portuguesa e espanhola, e 4) estar disponível gratuitamente. Apps que abordavam PIM não específicos para o tema, que focavam apenas em informações sobre medicamentos foram excluídos. Os apps relevantes foram visualizados em um dispositivo Android versão 10.0.0 ou em um dispositivo Apple versão 13.3.1 a fim de identificar se atenderam aos critérios de elegibilidade. Quando um aplicativo móvel foi encontrado nas duas lojas de apps, um dispositivo Android foi usado para fazer o download e posterior análises.

\section{Extração dos dados}

As principais informações dos apps incluídos foram extraídas, como: sistema operacional disponível, desenvolvedores, país, ano, número de atualizações, avaliações do usuário, tamanho (em MB), necessidade de conexão à internet e descrição geral.

\section{Avaliação da qualidade}

A qualidade dos apps foi avaliada utilizando o instrumento Mobile Application Rating Scale (MARS). ${ }^{(22)} \mathrm{O}$ instrumento MARS é uma escala de 23 itens composta de duas seções: qualidade objetiva com 19 itens, divididos em 4 seções e qualidade subjetiva com 4 itens. Os critérios da seção de qualidade objetiva do MARS tratam das seguintes propriedades: engajamento (5 itens), funcionalidade (4 itens), estética (3 itens) e qualidade da informação (7 itens). Cada item do instrumento possui uma descrição para classificação, exceto na seção de qualidade subjetiva, e pode ser avaliado em uma escala tipo Likert de 5 pontos ( 1 = inadequado, 2 = ruim, 3 = aceitável, 4 = bom e $5=$ excelente). Caso um item não seja adequado para um aplicativo, ele pode ser classificado como "não aplicável".

Para classificação dos apps, o guia de recomendação do instrumento MARS foi utilizado, bem como o vídeo de treinamento fornecido pelos seus desenvolvedores e disponível no 
YouTube (https://www.youtube.com/watch?v=25vBwJQIOcE). Uma discussão entre os autores foi realizada para alcançar um consenso sobre os critérios de classificação.

As quatro seções da qualidade objetiva foram calculadas de acordo a média dos itens classificados em cada seção. O índice geral de qualidade do aplicativo foi obtido pela média das quatro seções. A qualidade subjetiva foi avaliada separadamente, como itens individuais. Um ponto de corte de 3,0 foi estabelecido como uma pontuação mínima aceitável de qualidade. ${ }^{(22)}$ Apps com pontuações acima de 4,0 foram considerados de boa qualidade.

\section{RESULTADOS E DISCUSSÃO}

Pesquisa eletrônica

A pesquisa eletrônica identificou 252 aplicativos móveis potencialmente relevantes. Após a triagem de títulos e descrições nas lojas de apps, 19 deles foram baixados para triagem adicional. Destes, 5 apps atenderam aos critérios de inclusão e foram incluídos na revisão. Nenhum aplicativo relevante foi identificado por outras fontes. O fluxograma dos resultados da pesquisa dos apps está apresentado na Figura 1.

Figura 1: Fluxograma da seleção dos aplicativos móveis nas lojas Google Play Store e Apple Store.

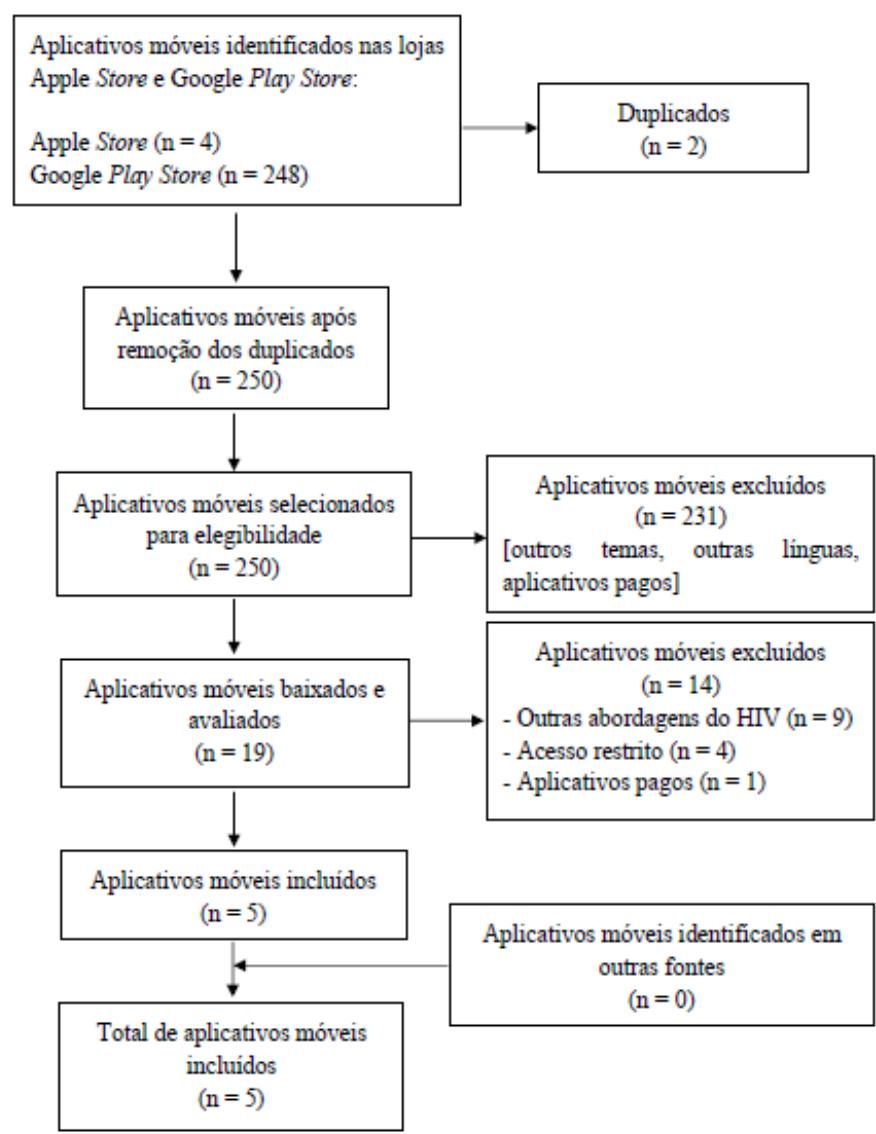

Fonte: Os autores. 
Características dos aplicativos móveis

A maioria dos apps estavam disponíveis apenas no Google Play Store (3/5, 60,0\%) e foram desenvolvidos na Espanha (2 apps), Inglaterra (1 app), Canadá (1 app) e Estados Unidos (1 app). Três apps estavam na língua inglesa e dois na língua espanhola. Três apps $(60,0 \%)$ foram atualizados nas lojas de apps nos últimos 2 anos. A avaliação média dos usuários (classificação por estrelas) dos aplicativos disponíveis na Google Play Store foi 4,35 (3,9 - 4,8) e na Apple Store foi 5,0, de um total de 5,0, sendo considerado uma ótima avaliação. O tamanho dos apps no sistema operacional iOs variou de 1,8 a 5,2 MB e no sistema Android variou de 3,1 a 19 MB. Nenhum dos apps possuem necessidade de conexão à internet para seu funcionamento, salvo para atualizações dos bancos de dados. A maioria dos aplicativos (4/5, $80 \%$ ) classificam os resultados obtidos das PIM em categorias e apenas um aplicativo fornece a classificação da qualidade de evidência. Dois apps (40,0\%) foram desenvolvidos por instituições hospitalares, seguido por universidade $(1 / 5,20,0 \%)$, organização não governamental $(1 / 5,20,0 \%)$ e comercial $(1 / 5,20,0 \%)$. Nenhum dos aplicativos móveis tiveram seus resultados publicados em revistas científicas revisada por pares. As características dos cinco apps incluídos no estudo estão apresentadas no Quadro 1.

Quadro 1. Características dos aplicativos móveis incluídos.

\begin{tabular}{|c|c|c|c|c|c|c|c|c|}
\hline Nome do app & $\begin{array}{c}\text { Sistema } \\
\text { operacional }\end{array}$ & Desenvolvedores & País & Versão/ano & $\begin{array}{l}\text { Avaliação } \\
\text { dos usuários }\end{array}$ & $\begin{array}{c}\text { Tamanho } \\
\text { (MB) }\end{array}$ & $\begin{array}{c}\text { Conexão } \\
\text { com internet }\end{array}$ & Descrição geral \\
\hline $\begin{array}{l}\text { Liverpool } \\
\text { HIV iChart }\end{array}$ & Android & $\begin{array}{l}\text { Universidade de } \\
\text { Liverpool }\end{array}$ & Inglaterra & $\begin{array}{l}2.0 .1 / \\
2018\end{array}$ & 5,0 & 4,7 & Não & $\begin{array}{l}\text { Mecanismo de busca de } \\
\text { PIM para verificar } \\
\text { interações entre } \\
\text { medicamentos para HIV } \\
\text { e outros medicamentos. } \\
\text { Os resultados da PIM } \\
\text { podem ser classificados } \\
\text { em cinco categorias: } \\
\text { vermelho (associação } \\
\text { contraindicada), laranja } \\
\text { (interação que pode } \\
\text { exigir ajuste da dose, } \\
\text { tempo de administração } \\
\text { e/ou monitoramento), } \\
\text { amarelo (interação } \\
\text { fraca), verde (interação } \\
\text { sem importância clínica) } \\
\text { e cinza (não há dados na } \\
\text { literatura). Um breve }\end{array}$ \\
\hline
\end{tabular}




\begin{tabular}{|c|c|c|c|c|c|c|c|c|}
\hline & & & & & & & & $\begin{array}{l}\text { resumo de } r \text { cada } \\
\text { interação é fornecido } \\
\text { juntamente com uma } \\
\text { classificação } \\
\text { qualidade da evidência } \\
\text { (muito baixa, baixa, } \\
\text { moderada, alta). }\end{array}$ \\
\hline $\begin{array}{l}\text { HIV-HCV } \\
\text { Drug Therapy } \\
\text { Guide }\end{array}$ & iOs & $\begin{array}{c}\text { Rede } \\
\text { Universitária de } \\
\text { Saúde - Hospitais }\end{array}$ & Canadá & $\begin{array}{l}1.0 .1 / \\
2017\end{array}$ & 5,0 & 1,8 & Não & 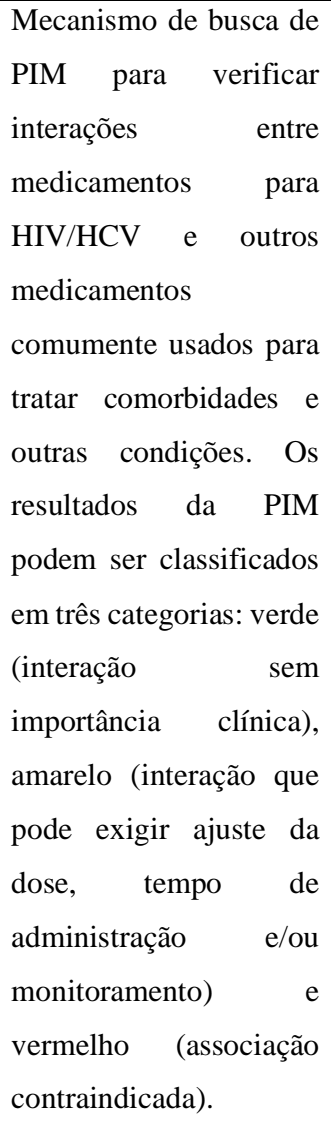 \\
\hline $\begin{array}{l}\text { HIV-Rx DDI } \\
\text { Check }\end{array}$ & Android & $\begin{array}{l}\text { VirologyEd } \\
\text { Consultants }\end{array}$ & $\begin{array}{l}\text { Estados } \\
\text { Unidos }\end{array}$ & $\begin{array}{l}1.1 .2 / \\
2019\end{array}$ & NR & 3,1 & Não & $\begin{array}{l}\text { Mecanismo de busca de } \\
\text { PIM para verificar } \\
\text { interações entre } \\
\text { medicamentos para HIV } \\
\text { e outros medicamentos. }\end{array}$ \\
\hline $\begin{array}{c}\text { Infodrogas_VI } \\
\mathrm{H}\end{array}$ & Android & $\begin{array}{c}\text { Grupo de } \\
\text { Trabalho para } \\
\text { Tratamento do } \\
\text { HIV }\end{array}$ & Espanha & $\begin{array}{l}1.0 .1 / \\
2018\end{array}$ & 4,8 & 4,5 & Não & $\begin{array}{l}\text { Mecanismo de busca de } \\
\text { PIM para verificar } \\
\text { interações entre } \\
\text { medicamentos para HIV } \\
\text { e outras substância de } \\
\text { uso recreativo e/ou } \\
\text { ilícita. Os resultados da } \\
\text { PIM podem ser } \\
\text { classificados em três } \\
\text { categorias: verde (risco } \\
\text { baixo), amarelo (risco }\end{array}$ \\
\hline
\end{tabular}




\begin{tabular}{|c|c|c|c|c|c|c|c|c|}
\hline & & & & & & & & $\begin{array}{l}\text { médio) e vermelho } \\
\text { (risco alto). }\end{array}$ \\
\hline $\begin{array}{c}\text { Interacciones } \\
\text { HIV }\end{array}$ & Android & $\begin{array}{l}\text { Hospital Clinic de } \\
\text { Barcelona }\end{array}$ & Espanha & $\begin{array}{l}2.2 .0 / \\
2017\end{array}$ & NR & 19,0 & Não & 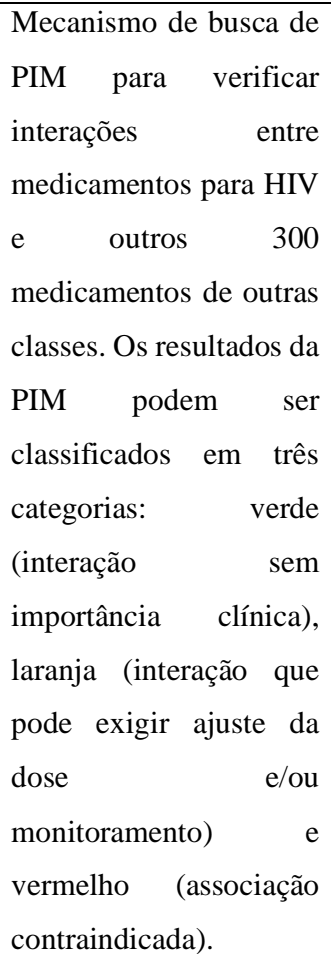 \\
\hline
\end{tabular}

Abreviação: App (aplicativo móvel), HCV (hepatite C), HIV (vírus da imunodeficiência humana), NR

(não reportado), PIM (potenciais interações medicamentosas).

Fonte: Os autores.

Avaliação da qualidade

As pontuações do MARS para os 5 aplicativos incluídos neste estudo estão apresentados na Figura 2. Além disso, as pontuações para cada aplicativo móvel da seção da qualidade objetiva, a classificação geral da qualidade objetiva e a seção da qualidade subjetiva do instrumento MARS são apresentadas na Tabela 1. A pontuação média total do instrumento MARS na seção da qualidade objetiva foi de 3,52 (DP 0,65). A pontuação média da seção da qualidade subjetiva foi menor que da seção da qualidade objetiva $(3,15$, DP 0,94). Apenas o aplicativo móvel ("Liverpool HIV iChart") alcançou uma pontuação geral maior que 4,0, indicando boa qualidade. 
Tabela 1. Avaliação da qualidade dos aplicativos móveis incluídos, segundo instrumento Mobile Application Rating Scale. ${ }^{22}$

\begin{tabular}{cccccccc}
\hline Nome do app & Engajamento & Funcionalidade & Estética & $\begin{array}{c}\text { Qualidade da } \\
\text { informação }\end{array}$ & $\begin{array}{c}\text { Pontuação geral } \\
\text { MARS }\end{array}$ & $\begin{array}{c}\text { Interpretação } \\
\text { da qualidade }\end{array}$ & $\begin{array}{c}\text { Qualidade } \\
\text { subjetiva }\end{array}$ \\
\hline $\begin{array}{c}\text { Liverpool HIV } \\
\text { iChart }\end{array}$ & 3,60 & 4,50 & 4,33 & 4,00 & 4,10 & Boa & 4,00 \\
$\begin{array}{c}\text { HIV-HCV Drug } \\
\text { Therapy Guide }\end{array}$ & 3,60 & 4,25 & 4,00 & 3,80 & 3,91 & Aceitável & 3,25 \\
$\begin{array}{c}\text { HIV-Rx DDI } \\
\text { Check }\end{array}$ & 2,80 & 2,75 & 2,66 & 3,20 & 2,85 & Ruim & 2,25 \\
$\begin{array}{l}\text { Infodrogas_VIH } \\
\text { Interacciones HIV }\end{array}$ & 3,60 & 4,50 & 4,00 & 3,80 & 3,97 & Aceitável & 3,50 \\
Média Total (DP) & $3,28(0,43)$ & $3,65(1,06)$ & $3,60(0,72)$ & $3,56(0,43)$ & $3,52(0,65)$ & Ruim & 2,50 \\
\hline
\end{tabular}

Abreviação: App (aplicativo móvel), MARS (Mobile Application Rating Scale), DP (desvio-padrão).

Fonte: Os autores.

Figura 2. Pontuação do instrumento Mobile Application Rating Scale (MARS) para os aplicativos incluídos.

Nota: O gráfico de radar mostra as pontuações médias do MARS. Cada seção foi avaliada em uma escala de 5 pontos, variando de 1 a 5 ( 1 = inadequado, $2=$ ruim, $3=$ aceitável, $4=$ bom e $5=$ excelente) .

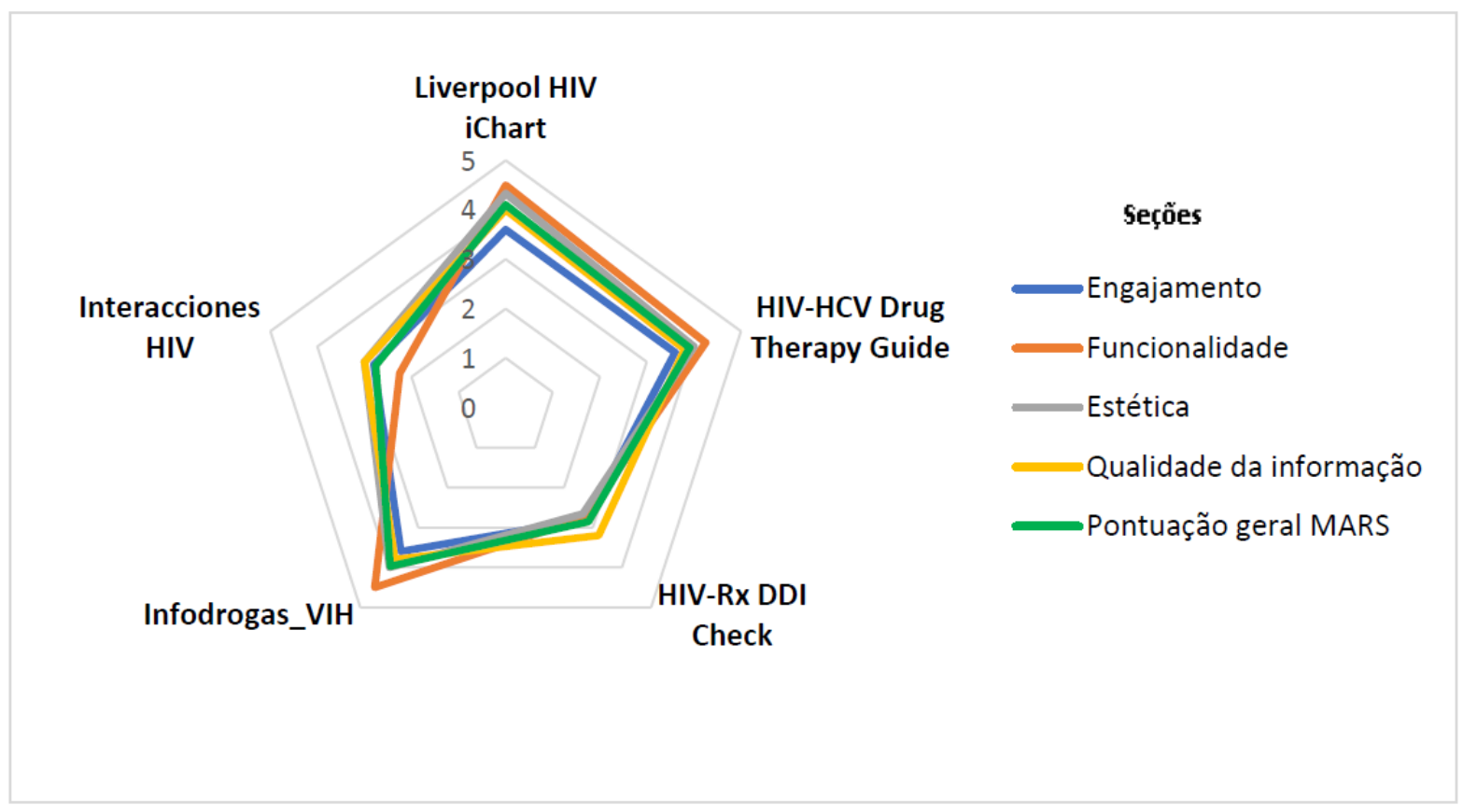

Fonte: Os autores.

Considerando as pontuações de cada seção da qualidade objetiva do instrumento MARS para apps incluídos, observou-se variabilidade nas classificações de engajamento, funcionalidade, estética e qualidade da informação. A seção Funcionalidade teve a maior 
pontuação média $(3,65$, DP 1,06$)$, sendo que a maioria dos apps $(3 / 5,60,0 \%)$ alcançou pontuações maiores que 4,0, indicando uma boa funcionalidade, especialmente pela facilidade do uso. As pontuações médias mais baixas foram para a seção Engajamento (3,28, DP 0,43), principalmente pela falta de interesse no uso dos apps. A seção Estética teve uma pontuação média de 3,60 (DP 0,72), sendo que a maioria dos aplicativos $(3 / 5,60,0 \%)$ alcançou pontuações máximas relacionadas ao conteúdo gráfico/resolução. Todos os apps atingiram pontuações aceitáveis $(\geq 3,00)$ na seção Qualidade da Informação, obtendo uma pontuação média de 3,56 (DP 0,43). Porém, é importante destacar que nenhum dos apps incluídos nesta revisão foi testado para avaliar sua evidência científica.

No geral, o app "Liverpool HIV iChart" obteve a maior pontuação geral do instrumento MARS $(4,10)$, seguido do “Infodrogas_VIH” $(3,97)$, "HIV-HCV Drug Therapy Guide” $(3,91)$, “HIV-Rx DDI Check" $(2,85)$ e "Interacciones HIV $(2,76)$ ”. Em relação a qualidade subjetiva, "Liverpool HIV iChart" alcançou a maior pontuação $(4,00)$, seguido de "Infodrogas_VIH" (3,50), "HIV-HCV Drug Therapy Guide" (3,25) e, "HIV-Rx DDI Check" $(2,25)$ e "Interacciones HIV" $(2,00)$.

Para nosso conhecimento, este é o primeiro estudo que identificou e avaliou a qualidade de aplicativos móveis para verificar PIM no tratamento de HIV/AIDS. Esta revisão incluiu cinco apps, fornecendo uma análise crítica das características e a qualidade de cada um utilizando o instrumento MARS.

Este estudo destaca lacunas que devem ser observadas nestes aplicativos. Embora todos os apps tenham alcançado pontuações satisfatórias na seção Qualidade da Informação, somente um deles declarou ser desenvolvido por uma universidade. Além disso, um app declararou o uso de recomendações ou guias de associações sobre interações medicamentosas no tratamento de HIV/AIDS e nenhum deles apresentou lista de referências da literatura científica. Ademais, a maioria dos apps não demonstrou entreterimento suficiente, dificultando a atenção e adesão do usuário.

Frente a estes resultados, recomenda-se o uso com cautela de alguns apps identificados nesta revisão pelos profissionais de saúde na sua prática clínica. É importante conferir se as informações contidas nestes apps são fidedignas e de qualidade. Por outro lado, as lojas de apps deveriam ser mais rigorosas com relação à disponibilidade de apps para a área de saúde. Ademais, os desenvolvedores de apps deveriam interagir com especialistas da área do HIV/AIDS na fase de elaboração do app para contribuir com o conteúdo científico. 
Nesta revisão, os resultados da busca foram maiores na Google Play Store, da mesma forma que a maioria dos apps incluídos estavam presentes nesta loja (4/5, 80,0\%). Diferenças entre as lojas de aplicativos podem explicar estes resultados. A criação de um app no sistema operacional Android é um processo mais simples do que um app no sistema iOS em termos de linguagens de programação. ${ }^{(23)}$ Soma-se a este fato que a disponibilização dos apps na Apple Store é mais cara (99 versus 29 dólares americanos) e mais demorada (duas semanas versus 24 horas) do que no Google Play Store. ${ }^{(23)}$ Ademais, o mecanismo de pesquisa do Google Play Store parece ser mais sensível que o da Apple Store, encontrando apps que não estavam associados ao tema desta revisão.

A maoria dos apps $(4 / 5,80,0 \%)$ tinham tamanhos, em MB, considerados pequenos. Um aplicativo pequeno possui uma vantagem de ocupar pouco espaço na memória do smartphone, melhorando a sua agilidade. ${ }^{(24)}$ Além disso, nenhum dos apps possui a necessidade de conexão à internet para o uso, o que facilita para os usuários com problemas de conexão com a Internet, principalmente em países em desenvolvimento, como o Brasil.

Em relação aos desenvolvedores, três apps $(3 / 5,60,0 \%)$ foram desenvolvidos por universidade e instituições hospitalares. Porém, nenhum deles descreve ou esclarece a fonte das informações contidas nos aplicativos. A falta de transparência na informação científica utilizada nos aplicativos se torna um perigo para sua utilização na prática clínica. ${ }^{(25)}$ Portanto, os usuários devem ter cuidado ao usá-los, uma vez que as informações contidas podem não ser confiáveis, uma vez que os desenvolvedores não citam as referências utilizadas na criação destes aplicativos.

De acordo com a avaliação do instrumento MARS, a maioria dos apps alcançou o mínimo de pontuação aceitável para cada seção, sendo o Engajamento a menor pontuação média entre os apps. Pontuações baixas nesta seção já foram descritas em outros estudos. ${ }^{(26,27,28)}$ A Seção de engajamento é avaliada com base nos itens: "entretenimento" (prazer no uso do aplicativo), “interesse" (interesse no uso do aplicativo), "personalização" (se o aplicativo permite que o usuário personalize configurações e preferências), "interatividade" (se o aplicativo permite a entrada do usuário, fornece feedback e contém avisos) e "público-alvo" (se o conteúdo - visuais, idioma e design - é apropriado para o público-alvo). ${ }^{(22)}$ Dois apps tiveram pontuações inadequadas (menor que 3,0) para os itens "entreterimento" e "interesse". O restante alcançou pontuções mínimas aceitáveis para ambos os itens. Esse achados sugerem que os desenvolvedores de apps deveriam envolver o usuário final durante a fase de desenvolvimento para melhorar estes critérios. 
Apesar da seção Funcionalidade ter recebido a pontuação média mais alta, dois apps obtiveram pontuações mínimas aceitáveis e um aplicativo obteve a menor pontuação possível para o item "desempenho" (relacionado a precisão e rapidez dos recursos do aplicativo), prejudicando a usabilidade destes apps. Por outro lado, a maioria deles obteve boas pontuações no item "apelo visual” (aparência do app e a consistência geral do aplicativo) da seção Estética, podendo aumentar a adesão ao app.

Sobre a seção Qualidade da Informação, todos os apps alcançaram receberam a pontuação mínima aceitável para os itens "qualidade das informações" (se o aplicativo tinha um conteúdo correto, bem escrito e relevante para o seu objetivo), "quantidade de informações" (se o aplicativo continha um conteúdo abrangente e conciso) e "credibilidade" (se a fonte está especificada na descrição na loja de apps ou no próprio aplicativo). Todos os apps foram classificados como "não aplicável" para os itens "informações visuais" e "com base em evidências científicas". Este resultado já era esperado para o item "informações visuais", uma vez que estes apps possuem somente informações escritas. Entretando, o item "com base em evidências científicas" foi classificado desta forma uma vez que nenhum aplicativo incluído neste estudo foi testado e publicado em revistas científicas.

Como mencionado anteriormente, a pontuação da seção da qualidade subjetiva foi menor que a da qualidade objetiva, sendo semelhante a outros estudos. ${ }^{(28,29)}$ Além disso, a maioria dos apps obteve a pontuação mais baixa (não) no item “Você pagaria por este aplicativo?”. Isso é esperado já que todos os aplicativos incluídos nesta revisão são gratuitos. A intenção de pagar por um aplicativo deve levar em consideração um bom serviço disponível, com diferentes opções de personalização e com uma maior relação entre apelo visual e desempenho. ${ }^{(29)}$ É importante observar que essas pontuações têm a natureza subjetiva, sem afetar a pontuação geral de qualidade do MARS.

Esta revisão apresenta algumas limitações. É possível que alguns apps tenham sido perdidos na busca, uma vez que foram excluídos aplicados pagos ou que não estavam disponíveis nos sistemas operacionais Android ou iOs, embora estes sistemas representaram aproximadamente $99 \%$ da participação de mercado de telefonia móvel em 2019. ${ }^{(30)}$ Além disso, o mercado de apps é altamente dinâmico e a disponibilidade de aplicativos muda regularmente. Por fim, a busca por estes apps foi limitada às lojas de apps brasileiras, sendo que estas lojas permitem que os editores restrinjam as distribuições a países específicos, embora a maioria dos apps seja lançada em todo o mundo e não seja específica de um país. 


\section{CONCLUSÃO}

A maioria dos apps foram desenvolvidos por instituições hospitalares e universidade e classificam os resultados encontrados das PIM em categorias. Três apps apresentaram pontuações gerais aceitáveis do instrumento MARS, porém apenas um foi avaliado com boa qualidade $(>4,0)$. A seção Funcionalidade recebeu a maior pontuação dos apps identificados, enquanto a seção Engajamento obteve a menor pontuação. Assim, as falhas observadas nesta revisão mostram que estes apps devem ser utilizados com cautela pelos profissionais da saúde, bem como há necessidade de envolver o usuário final na atualização ou desenvolvimento de novos apps sobre o tema.

\section{REFERÊNCIAS BIBLIOGÁFICAS}

1. World Health Organization. Preventing chronic diseases: a vital investment: WHO global report. Geneva; 2015.

2. Hajata C, Steinb E. The global burden of multiple chronic conditions: A narrative review. Prev Med Rep 2018;12:284-293.

3. Brasil. Ministério da Saúde. Secretaria de Vigilância em Saúde. Departamento de Vigilância, Prevenção e Controle das Infecções Sexualmente Transmissíveis, do HIV/AIDS e das Hepatites Virais. Protocolo Clínico e Diretrizes Terapêuticas para Manejo da Infecção pelo HIV em Adultos. Brasília; 2018.

4. World Health Organization. HIV/AIDS Key facts. Disponível em: https://www.who.int/news-room/fact-sheets/detail/hiv-aids

5. World Health Organization. Global health sector strategy on HIV, 2016-2021. Geneva; 2019.

6. Osorio LT, Rivera MC, Marín DEP, Giraldo NA, Amariles P. Relevancia clínica de las interacciones medicamentosas en pacientes infectados con el virus de la inmunodeficiencia humana: actualización 2015-2017. Rev Chilena Infectol 2019;36:475-489.

7. Geocze L, Mucci S, Marco MA, Nogueira-Martins LA, Citero VA. Qualidade de vida e adesão ao tratamento anti-retroviral de pacientes portadores de HIV. Rev Saúde Pública 2010;44:743-9.

8. Gutierrez MM, Mateo MG, Corbacho N, Vidal F, Domingo P. Drug-drug interactions when treating HIV-related metabolic disorders. Expert Opin Drug Metab Toxicol 2019:15:787-802.

9. Furini AAC, Lima TAM, Rodrigues JF, Borges MSB, Carmo EGB, Cecchim MC, et al. Análise de interações medicamentosas e alimentares em pacientes com AIDS em uso da TARV associada à terapia de resgate. Rev Ciênc Farm Básica Apl 2015;36:427-434.

10. Dionne B. Key Principles of Antiretroviral Pharmacology. Infect Dis Clin N Am 2019;33:787-805.

11. Santos WM, Secoli SR, Padoin SMM. Potenciais interações de drogas em pacientes de terapia antirretroviral. Rev. Latino-Am. Enfermagem 2016;24:e2832

12. Baig MM, GholamHosseini H, Connolly MJ. Mobile healthcare applications: system design review, critical issues and challenges. Australas Phys Eng Sci Med 2015;38:23-38. 
13. Tibes CMS, Dias JD, Zem-Mascarenhas SH. Aplicativos móveis desenvolvidos para a área da saúde no Brasil: revisão integrativa da literatura. Rev Min Enferm 2014:18;471-478.

14. Koehler N, Vujovic O, McMenamin C. Healthcare professionals' use of mobile phones and the internet in clinical practice. J Mob Technol Med 2013:2;3-13.

15. Hofer F, Haluza D. Are Austrian practitioners ready to use medical apps? Results of a validation study. BMC Med Inform Decis Mak 2019:19;1-9.

16. Herron J. Bad Apps: mHealth Apps Doubling as Medical Devices. J Electron Resour Med Libr 2016:13;177-181.

17. Boulos MNK, Brewer AC, Karimkhani C, Buller DB, Dellavalle RP. Mobile medical and health apps: state of the art, concerns, regulatory control and certification. Online J Public Health Inform 2014;5:e229.

18. Jupp JCY, Sultani H, Cooper CA, Peterson KA, Truong TH. Evaluation of mobile phone applications to support medication adherence and symptom management in oncology patients. Pediatr Blood Cancer 2018;65:e272-278.

19. Apidi NA, Muthuveloo R, Caruso V, Chiau L. Mobile Medical Applications for Dosage Recommendation, Drug Adverse Reaction, and Drug Interaction: Review and Comparison. Ther Innov Regul Sci 2017;51: 480-485.

20. Jamaladin H, van de Belt TH, et al. Mobile Apps for Blood Pressure Monitoring: Systematic Search in App Stores and Content Analysis. JMIR Mhealth Uhealth 2018;6:e187.

21. Escoffery C, McGee R, Bidwell J, Sims C, Thropp EK, Frazier C, et al. A review of mobile apps for epilepsy self-management. Epilepsy Behav 81;2018:62-69.

22. Stoyanov SR, Hides L, Kavanagh DJ, Zelenko O, Tjondronegoro D, Mani M. Mobile App Rating Scale:A New Tool for Assessing the Quality of Health Mobile Apps. JMIR Mhealth Uhealth 2015;3(1):e27.

23. Tracy KW. Mobile application development experiences on Apple's iOS and Android OS. IEEE Potentials 2012;31:30-34.

24. Virani A, Duffett-Leger L, Letourneau N. Parenting apps review: in search of good quality apps. mHealth 2019;5:44-59.

25. Buijink AWG, Visser BJ, Marshall L. Medical apps for smartphones: lack of evidence undermines quality and safety. Evid Based Med 2013;18:90-92.

26. Tofighi B, Chemi C, Ruiz-Valcarcel J, Hein P, Hu L. Smartphone Apps Targeting Alcohol and Illicit Substance Use: Systematic Search in in Commercial App Stores and Critical Content Analysis. JMIR Mhealth Uhealth 2019;7:e11831.

27. Li Y, Ding J, Wang Y, Tang C, Zhang P. Nutrition-Related Mobile Apps in the China App Store: Assessment of Functionality and Quality. JMIR Mhealth Uhealth 2019;7:e13261.

28. Jovičić S, Siodmiak J, Watson ID. Quality evaluation of smartphone applications for laboratory medicine. Clin Chem Lab Med 2019;57:388-397.

29. Rodrigues AT, Sousa CT, Pereira J, Figueiredo IV, Lima TM. Mobile Applications (Apps) to Support the Hepatitis C Treatment: A Systematic Search in App Stores. Ther Innov Regul Sci 2020.

30. GlobalStat. StatCounter [cited 2019 Nov 8]. Disponível em: https://gs.statcounter.com/osmarket-share/mobile/worldwide. 